\title{
Effects of Adjuvant Mental Practice on Affected Upper Limb Function Following a Stroke: Results of Three-Dimensional Motion Analysis, Fugl-Meyer Assessment of the Upper Extremity and Motor Activity Logs
} Hyun Seung Oh, MD' ${ }^{1}$, Eun Joo Kim, MD', Doo Young Kim, $\mathrm{MD}^{2}$, Soo Jeong Kim, $\mathrm{MD}^{1}$

${ }^{1}$ Department of Rehabilitation Medicine, National Rehabilitation Center, Seoul;

${ }^{2}$ Goseong Community Health Center, Gyeongsangnam-do, Korea

\begin{abstract}
Objective To investigate the effects of adjuvant mental practice (MP) on affected upper limb function following a stroke using three-dimensional (3D) motion analysis.

Methods In this AB/BA crossover study, we studied 10 hemiplegic patients who had a stroke within the past 6 months. The patients were randomly allocated to two groups: one group received MP combined with conventional rehabilitation therapy for the first 3 weeks followed by conventional rehabilitation therapy alone for the final 3 weeks; the other group received the same therapy but in reverse order. The MP tasks included drinking from a cup and opening a door. MP was individually administered for 20 minutes, 3 days a week for 3 weeks. To assess the tasks, we used 3D motion analysis and three additional tests: the Fugl-Meyer Assessment of the upper extremity (FMAUE) and the motor activity logs for amount of use (MAL-AOU) and quality of movement (MAL-QOM). Assessments were performed immediately before treatment (T0), 3 weeks into treatment (T1), and 6 weeks into treatment (T2).

Results Based on the results of the 3D motion analysis and the FMA-UE index ( $\mathrm{p}=0.106)$, the MAL-AOU scale ( $\mathrm{p}=0.092)$, and MAL-QOM scale ( $\mathrm{p}=0.273$ ), adjuvant MP did not result in significant improvements.

Conclusion Adjuvant MP had no significant effect on upper limb function following a stroke, according to 3D motion analysis and three clinical assessment tools (the FMA-UE index and the two MAL scales). The importance of this study is its use of objective 3D motion analysis to evaluate the effects of MP. Further studies will be needed to validate these findings.
\end{abstract}

\section{Keywords Upper extremity, Stroke}

Department of Rehabilitation Medicine, National Rehabilitation Center, 58 Samgaksan-ro, Gangbuk-gu, Seoul 01022, Korea. Tel: +82-2-901-1633, Fax: +82-2-901-3835, E-mail: silverzookim@gmail.com

ORCID: Hyun Seung Oh (http://orcid.org/0000-0002-9765-0397); Eun Joo Kim (http://orcid.org/0000-0001-6166-7886); Doo Young Kim (http://orcid. org/0000-0003-1327-5348); Soo Jeong Kim (http://orcid.org/0000-0003-3951-6947).

(c) This is an open-access article distributed under the terms of the Creative Commons Attribution Non-Commercial License (http://creativecommons.org/ licenses/by-nc/4.0) which permits unrestricted noncommercial use, distribution, and reproduction in any medium, provided the original work is properly cited. Copyright $\odot 2016$ by Korean Academy of Rehabilitation Medicine 


\section{INTRODUCTION}

Stroke patients with dysfunction of the upper extremities can face significant problems in their activities of daily living (ADLs) as well as in the recovery of other general functions [1].

Although many different therapeutic approaches are available for improving upper extremity function after a stroke [1], it is important to select the most appropriate intervention for rehabilitation in accordance with the severity of impairment.

Mental imagery is an active process that combines all six senses: visual, auditory, tactile, kinesthetic, olfactory, and gustatory [2]. Motor imagery, a component of mental imagery, is associated with a specific movement produced by the internal reproduction of motor action without motor output [2,3]. Mental practice (MP) involves motor imagery and includes repetitive imagination of a physical activity with the intention of performing that activity or improving performance $[2,4]$. MP allows an individual to perform tasks repeatedly without physical exhaustion or any risk to safety [5]. In addition, it enables patients to practice complex physical tasks that the stroke had rendered difficult.

MP was first used in sports to improve techniques, and it is believed that neural loops and movement patterns may be activated during MP [1]. The application of MP in stroke patients was reported to activate the cerebral and cerebellar sensorimotor structures repeatedly [6], and similar results were obtained when the actual tasks were practiced, according to a study involving positron emission tomography [7]. Another study [8] showed that MP increased activity in the premotor area, the primary motor cortex, and the superior parietal cortex. In patients receiving hemiplegic stroke rehabilitation, the application of MP along with other neurological practices was shown to help recovery of unilateral upper limb function at a low cost and without risks or complications [8-10].

Based on a review of the Cochrane database in 2011 (6 trials, $n=119$ ), the use of rehabilitation treatments combined with MP was found to be more effective for improving upper extremity function after stroke than were rehabilitation treatments without MP [4]. Previous studies assessed MP for accomplishing ADLs (such as ironing or buttoning a shirt, turning a page in a book, lifting a cup, or opening a door). However, results of several studies using the Fugl-Meyer Assessment of the upper extremity (FMA-UE), the action research arm test (ARAT), and the motor activity log (MAL) to evaluate muscle power and hand function indicated a mismatch between the intervention and evaluation methods [10-12].

Conventional studies $[10,11,13,14]$ have shown that upper extremity function can be improved with adjunctive MP; however, in these studies, the tasks performed during MP and the tools used for evaluating upper extremity function differed, making it difficult to measure the actual changes. Furthermore, the authors of a previous study [12] claimed that patients with motor recovery after a stroke episode that occurred within the previous 6 months (subacute) did not benefit from MP. These patients had performed tasks such as opening, grasping, and lifting household objects; however, upper extremity function was measured by means of the ARAT, which led to differences between the tasks and the evaluation method. In order to evaluate the actual changes in a patient's motions, we used objective three-dimensional (3D) motion analysis to investigate the identical motions that correspond to MP (in this case, drinking from a cup and opening a door).

The patients assessed in previous MP studies usually had chronic stroke, and few such studies have been performed in patients with subacute stroke. Because our hospital treats mainly those with subacute stroke, we focused on the effects of MP in this group.

In order to participate in therapy and follow instructions, patients undergoing traditional studies of MP and upper limb function $[1,8,10-13,15,16]$ are required to have good cognitive scores on the Mini-Mental State Examination (MMSE) or stable mental status, as well as the ability to understand verbal instructions. However, adequate MMSE scores and compliance with instructions alone are not sufficient to validate the effectiveness of MP. Therefore, our investigation cites studies on motor imagery $[17,18]$, evaluating patients using a standard score of 2.26 on the Vividness of Movement Imagery Questionnaire (VMIQ).

In the present study, additional MP was provided to stroke patients who practiced conventional occupational therapy and performed identical tasks along with MP. Moreover, 3D motion analysis was carried out to understand the effects of MP on upper extremity function in real life after a stroke. We also compared the outcomes of 
3D motion analysis and of clinical assessments (FMA-UE and MAL) to detect evidence of any congruity between these methods of evaluation.

\section{MATERIALS AND METHODS}

\section{Subjects}

Initially, 15 stroke patients were enrolled, but during the study 5 patients withdrew their consent to participate. The study included 10 stroke patients who were admitted to our hospital between March and November 2012. The patients had mean scores of 28.3 on the Korean version of the Mini-Mental State Examination (K-MMSE), 68 on the Korean version of the Modified Barthel Index (K-MBI), and 1.53 on the VMIQ at an average of 128.1 days from stroke onset. Of the 10 patients, 7 were diagnosed with infarction and 3 with hemorrhage (Table 1).

The inclusion criteria were as follows: (1) infarction or hemorrhage as the primary diagnosis; (2) hemiplegia with unilateral lesions; (3) subacute status, meaning that the stroke occurred more than 1 month before the study began but within the previous 6 months; (4) the ability to lift a cup and open a door using the impaired extremity, with a rating of stage $>3$ on the Brunnstrom scale (to permit upper extremity motion analysis); (5) K-MMSE scores of $>24$ (to be able to understand and follow instructions during the MP study); and (6) adequate degree of imagination for MP (i.e., patients who score low on the

Table 1. General characteristics of the subjects $(n=10)$

\begin{tabular}{lc}
\hline \multicolumn{1}{c}{ Characteristic } & Value \\
\hline Age (yr) & $57.9 \pm 15.47$ \\
Sex (male:female) & $7: 3$ \\
Days from onset of stroke & $128.1 \pm 26.05$ \\
Brain hemisphere affected (right:left) & $4: 6$ \\
Diagnosis (infarction:hemorrhage) & $7: 3$ \\
Brunnstrom stage for arm & $4.3 \pm 1.25$ \\
K-MMSE & $28.3 \pm 1.95$ \\
K-MBI & $68 \pm 16.83$ \\
VMIQ & $1.53 \pm 0.45$ \\
\hline
\end{tabular}

Values are presented as mean \pm standard deviation or number.

K-MMSE, Korean version of the Mini-Mental State Examination; K-MBI, Korean version of Modified Barthel Index; VMIQ, Vividness of Movement Imagery Questionnaire.
VMIQ are effectively able to use motor imagery for movement of the upper extremity) $[17,18]$. Exclusion criteria were as follows: (1) any surgery within 6 months prior to the study; (2) fractures or musculoskeletal injuries on the hemiplegic side; and (3) severe hemiplegic spasticity, with a Modified Ashworth Scale (MAS) score of $\geq 3$, or severe pain, with a score of $\geq 5$ on the visual analog scale (VAS). All patients were required to sign an informed consent form that was approved by the Institutional Review Board of the Korea National Rehabilitation Center.

\section{Study protocol}

We used an AB/BA crossover design for this study. After providing informed consent, the patients were divided into two groups of five patients each. A computer-generated system was used for randomization, and the assignment of patients to the groups was performed using a randomly permuted block design, with a block size of 4. Group 1 performed MP for 20 minutes, 3 times a week, in addition to conventional rehabilitation therapy for 30 minutes, 5 times a week, for the first 3 weeks. This was followed by 3 weeks of conventional rehabilitation therapy alone. Group 2 received only the conventional rehabilitation therapy for the first 3 weeks, followed by MP for 20 minutes, 3 times a week, in addition to conventional rehabilitation therapy for 30 minutes, 5 times a week for the following 3 weeks, for a total of 6 weeks (Fig. 1).

The MP protocol included two tasks-drinking from a cup and opening a door-and included three, 20-minute steps: relaxation, MP, and refocusing. Patients were placed in a quiet, comfortable room and listened to a pre-recorded script directing them to imagine that they were moving the affected upper extremity.

The evaluation of the affected upper extremity involved 3D motion analysis of the two tasks to determine the kinematic factors involved in shoulder, elbow, and wrist motions (Fig. 2). According to a previous study, 3D motion analysis can be used to quantify upper extremity motion and to help in assessing the outcomes of stroke rehabilitation [19]. Another study reported that 3D motion analysis can be used to objectively quantify upper extremity motor performance during functional tasks in patients who can perform the tasks of reaching and drinking [20]. We used the Vicon MX-T20 motion analysis system (Vicon Motion Systems, Oxford, UK) to evaluate the angle of each joint and changes in position. With this 

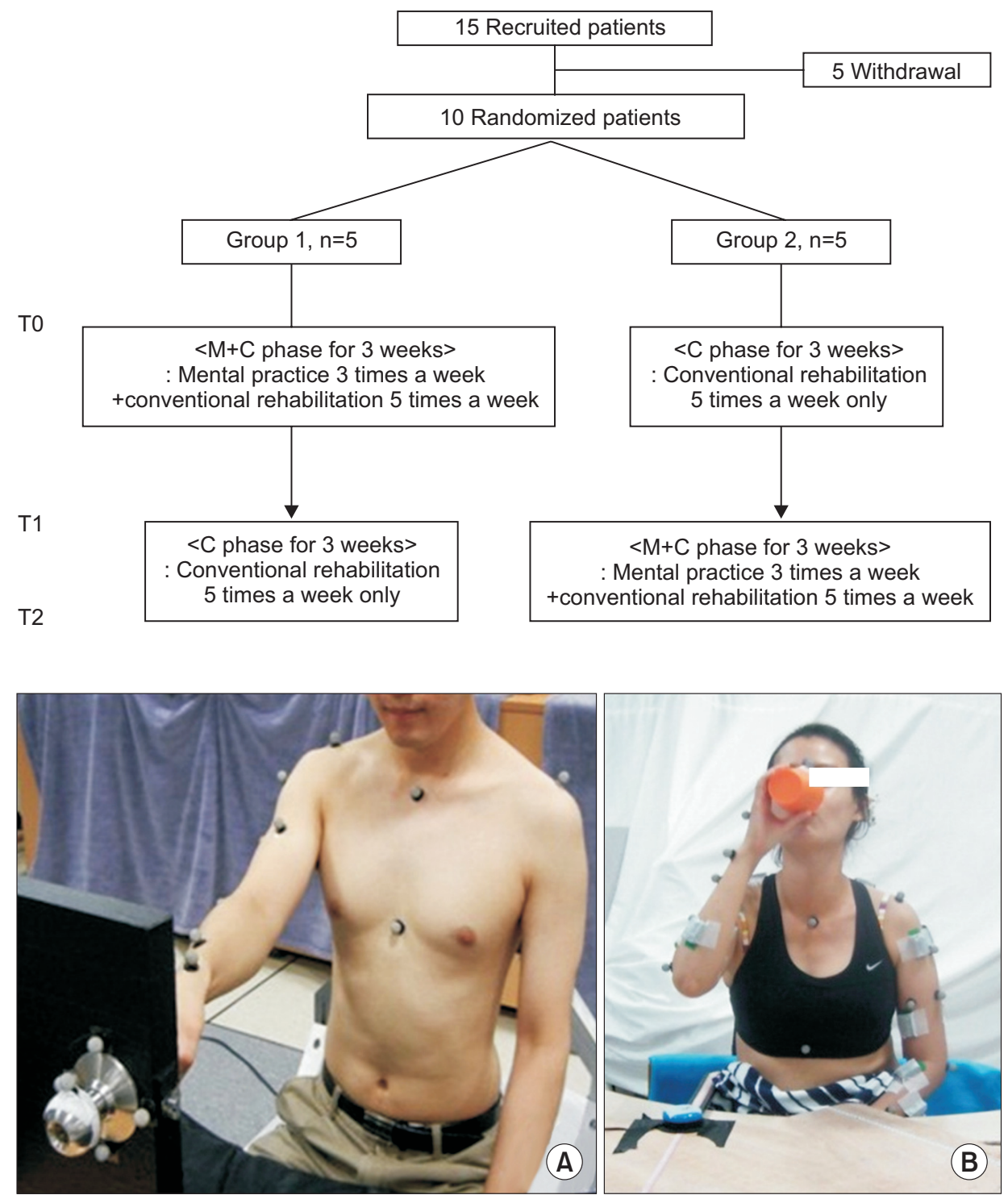

Fig. 1. Crossover study design, including three phases: T0 was the first assessment, performed at the start; T1 was the second assessment, performed at phase-change; and $\mathrm{T} 2$ was the third assessment, performed at the end.

Fig. 2. Images of the three-dimensional motion analysis using the Vicon MX-T20 system for opening a door and drinking from a cup. (A) Opening a door and (B) drinking from a cup. system, eight cameras (sampling at $200 \mathrm{~Hz}$ ) were used to track the 3D trajectories of reflective markers placed on the skin.

For the tasks involving drinking from a cup, the patients were seated comfortably in a chair, and the cup was placed on a table in front of them. They were then asked pick up the cup, drink from it, and put it back on the table. Changes in circumduction ratio, velocity, smoothness, elapsed time, and joint angle were measured, and the analysis included six phases: (1) the reaction phase (between the beginning of the experiment and before the initial movement of the body); (2) the reaching phase (when the hand stretches out for the cup); (3) the forward transfer phase (when the patient brings the cup to his or her mouth); (4) the drinking phase; (5) the back transfer phase (when the patient places the cup back down); and (6) the returning phase (when the patient's hand is withdrawn from the cup). In addition, the joints of the upper arm were divided for analysis into the shoulder, elbow, wrist, and fingers.

For tasks involving opening a door, the patients were instructed to take hold of the doorknob, turn it, and then return it to its original position. In this experiment, changes in velocity, smoothness, elapsed time, and joint angle were measured. Motion was evaluated during two phases: supination and pronation. Velocity, smoothness, elapsed time, and each joint angle were evaluated as factors of motion analysis, and the FMA-UE and MAL scales were used. All evaluations were performed three times: before treatment (T0), 3 weeks into treatment (T1), and 6 
weeks into treatment (T2).

\section{D motion analysis factors}

We measured the following factors for the motion analysis: reaction time, movement time, peak velocity (PV), peak angle velocity, range of motion (ROM), normalized jerk score (NJS), interjoint coordination, straightness ratio (SR), and movement units.

\section{Clinical assessment of motor function}

For clinical assessment of the results, we used the FMAUE and MAL scales.

\section{Sample size}

The G*Power program was used to yield a sample size of the test subjects (G*Power v3.0.10; Franz Faul, Kiel University, Germany). In addition, $G^{*}$ Power was used to yield the sample size for the amount of use (AOU) and quality of movement (QOM) scales of the MAL tests [16]. For the AOU scale of the MAL test, an $\alpha$-error of 0.05 , a $\beta$ of 0.2 (power, $80 \%$ ), and an effect size of 2.4 were the settings in the calculation using $\mathrm{G}^{*}$ Power. For the QOM scale of the MAL test, an $\alpha$-error of 0.05 , a $\beta$ of 0.2 (power, $80 \%$ ), and an effect size of 4.0 were the settings in the calculation using $\mathrm{G}^{*}$ Power. The results showed that the control and experimental groups required at least 5 patients each. Ultimately, considering the dropout rate, 15 patients were recruited.

\section{Statistical analysis}

To compare the effect of each intervention (adjuvant MP vs. conventional rehabilitation alone), a pre-analysis process was used because of the crossover study design. We assessed the homogeneity of the initial values, the period effect, and the treatment-period interaction between the two groups (Group 1 vs. Group 2) (Fig. 1).

To test and compare the homogeneity of the initial values (T0) between the two groups, the Mann-Whitney U test was used. Prior to the evaluation (T0), the motion analysis factors and the FMA-UE and MAL results were compared between the groups, and no significant differences were noted; therefore, homogeneity could be assessed.

The period effect was compared between the two groups using the Mann-Whitney U test, which was also used to assess differences in the evaluation scores be- fore and after MP, the motion analysis factors of the two groups, and the FMA-UE and MAL results. To study the effect of time in both the groups, we compared differences in the FMA-UE and MAL results and the 3D motion analysis factors between the two groups during each $\mathrm{M}+\mathrm{C}$ phase (MP plus conventional rehabilitation). However, no significant differences were noted between the two groups, indicating that time had no impact on the effectiveness of the treatments.

We investigated the possibility of a treatment-period interaction by assessing whether in the absence of an interaction, a patient's average response to the treatments would be the same, regardless of the order in which they were received. For the motion analysis factors and the FMA-UE and MAL results of the two groups, the MannWhitney U test was used to assess differences between the evaluation scores before treatment and after 6 weeks of treatment. However, no significant change was noted in the scores of either group, indicating that the sequence of the tests had no impact on the effectiveness of the treatment with respect to the treatment-period interaction.

Thus, we found no significant differences between the two groups with respect to initial values, period effect, or treatment-period interaction. Therefore, to study the effects of MP, the results could be analyzed after regrouping the patients into Group M (MP combined with conventional rehabilitation therapy) and Group C (conventional rehabilitation therapy alone) (Fig. 1). Consequently, we based the verification of the effects of MP on the scores of a group of 10 patients before and after carrying out both conventional therapy and MP, and we compared the findings with those of another group of patients who underwent conventional therapy only. The Wilcoxon test was used to compare the assessments before and after training. All statistical analyses were performed using SPSS ver. 14.0 software for Windows (SPSS Inc., Chicago, IL, USA). Differences with a p-value $<0.05$ were considered statistically significant.

\section{RESULTS}

Assessment of period effect and treatment-period interaction

To investigate the period effect in both groups, we compared differences in the FMA-UE and MAL results 
and the 3D motion analysis factors between Group 1 and Group 2 during each $\mathrm{M}+\mathrm{C}$ phase. We found no significant differences between the two groups in the following parameters: FMA-UE ( $p=0.151)$, MAL-AOU $(p=0.151)$, MAL-QOM ( $\mathrm{p}=0.690)$, drinking from a cup reaction time ( $\mathrm{p}=0.151)$, movement time $(\mathrm{p}=0.548)$, total PV $(\mathrm{p}=0.548)$, total NJS ( $\mathrm{p}=0.151)$, total SR $(\mathrm{p}=0.730)$, total movement unit $(\mathrm{p}=0.548)$, opening a door reaction time $(\mathrm{p}=0.056)$, total movement time $(\mathrm{p}=0.548)$, pronation PV $(\mathrm{p}=1.000)$, supination PV ( $\mathrm{p}=1.000)$, and NJS pronation $(\mathrm{p}=0.548)$.
These results showed that time had no impact on the effectiveness of the treatments.

To investigate the possibility of a treatment-period interaction, we compared differences in the FMA-UE and MAL results and 3D motion analysis factors before the treatment and after 6 weeks of treatment between Group 1 and Group 2. We found no significant differences between the two groups in the following parameters: FMAUE ( $\mathrm{p}=0.690)$, MAL-AOU ( $\mathrm{p}=0.548)$, MAL-QOM ( $\mathrm{p}=1.000)$, drinking from a cup reaction time $(\mathrm{p}=0.095)$, movement
(A)

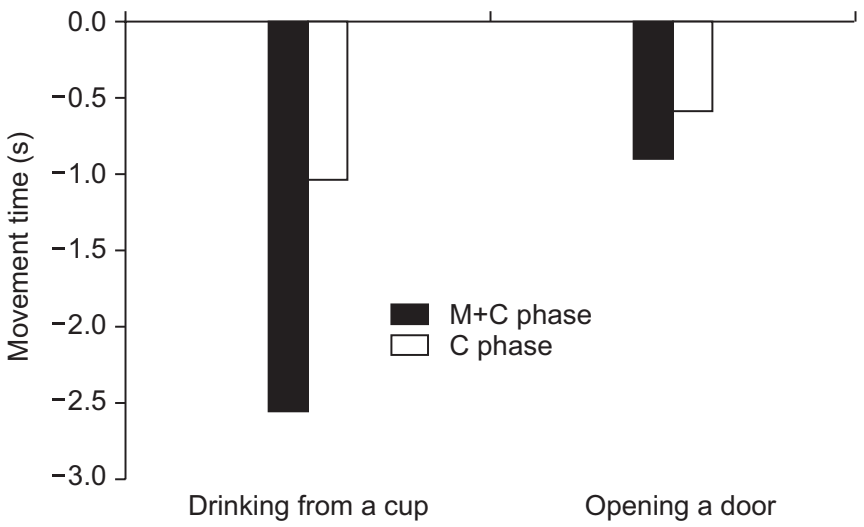

(B)

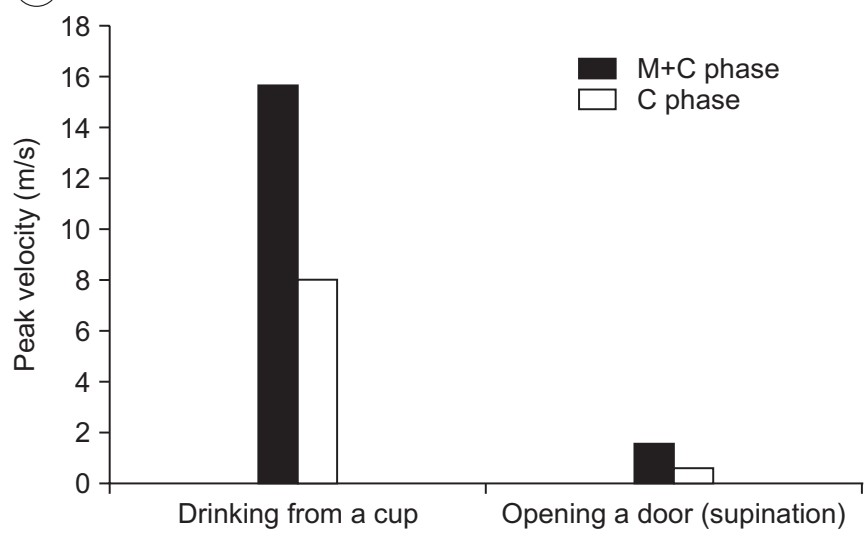

Fig. 3. Comparison of three-dimensional motion analysis data between the $M+C$ phase and the $C$ phase. (A) Comparison of movement time and (B) comparison of peak velocity. $\mathrm{M}+\mathrm{C}$ phase, mental practice and conventional therapy phase; $\mathrm{C}$ phase, conventional therapy phase only.

Table 2. Comparison of results of 3D motion analysis by task between the $\mathrm{M}+\mathrm{C}$ phase and the $\mathrm{C}$ phase

\begin{tabular}{|cccc|}
\hline 3D motion analysis & M+C phase & C phase & p-value \\
\hline Drinking from a cup & & & \\
\hline Reaction time (s) & $-0.056 \pm 0.176$ & $-0.204 \pm 0.540$ & 0.285 \\
\hline Movement time $(\mathrm{s})$ & $-2.560 \pm 1.970$ & $-1.033 \pm 1.640$ & 0.139 \\
\hline PV (m/s) & $15.671 \pm 11.903$ & $8.015 \pm 11.675$ & 0.169 \\
\hline NJS $\left(\mathrm{m} / \mathrm{s}^{3}\right)$ & $-3,295.14 \pm 9,040.27$ & $-22,190.80 \pm 45,186.76$ & 0.138 \\
\hline MU (m/s $\mathrm{s}^{3}$ & $-3.000 \pm 6.624$ & $-8.142 \pm 8.551$ & 0.167 \\
\hline Opening a door & & & \\
\hline Reaction time $(\mathrm{s})$ & $0.028 \pm 0.074$ & $-0.036 \pm 0.099$ & 0.152 \\
\hline Movement time $(\mathrm{s})$ & $-0.887 \pm 0.800$ & $-0.586 \pm 0.786$ & 0.333 \\
\hline Pronation PV $(\mathrm{m} / \mathrm{s})$ & $1.321 \pm 9.396$ & $1.392 \pm 10.095$ & 0.799 \\
\hline Supination PV $(\mathrm{m} / \mathrm{s})$ & $1.536 \pm 8.883$ & $0.662 \pm 9.358$ & 0.721 \\
\hline NJS $\left(\mathrm{m} / \mathrm{s}^{3}\right)$ & $3,338.24 \pm 35,459.87$ & $-3,109.41 \pm 4,015.28$ & 0.799 \\
\hline
\end{tabular}

Values are presented as mean \pm standard deviation and were derived by subtracting the values at the end of each phase from those at the start (T2 - T1, T1 - T0).

$\mathrm{M}+\mathrm{C}$ phase, mental practice and conventional therapy phase; C phase, conventional therapy phase only; PV, peak velocity; NJS, normalized jerk score; MU, movement unit. 
time ( $\mathrm{p}=0.222)$, total PV $(\mathrm{p}=0.310)$, total NJS $(\mathrm{p}=0.222)$, total SR ( $\mathrm{p}=0.841)$, total movement unit $(\mathrm{p}=0.151)$, opening a door reaction time $(\mathrm{p}=0.841)$, total movement time $(\mathrm{p}=1.000)$, pronation $\mathrm{PV}(\mathrm{p}=0.690)$, supination PV $(\mathrm{p}=0.222)$, and NJS pronation $(\mathrm{p}=0.222)$. These results showed that the sequence of the tests had no impact on the effectiveness of the treatments with respect to treatment-period interaction.

\section{Comparison of Group M and Group C with respect to $3 \mathrm{D}$ motion analysis}

In the tasks that involved drinking from a cup and opening a door, the 3D motion analysis showed that movement time did not significantly decrease when MP has been performed (Fig. 3A). In addition, the peak velocity in drinking from a cup and the supination peak velocity in opening a door showed no significant improvement (Fig. 3B); however, the two groups showed no significant differences for these values (Table 2). Although the pre- and post-treatment effects showed improvements, the differences were not statistically significant on the 3D motion analysis. The results of MP combined with conventional rehabilitation therapy and of conventional rehabilitation therapy alone did not differ significantly on
3D motion analysis.

\section{Comparison of Group M and Group C with respect to} the FMA-UE and MAL scales

Table 3 presents the changes in each patient's FMAUE results when MP and conventional therapy were performed simultaneously. On comparing the FMA-UE results between the two groups, we found that functional

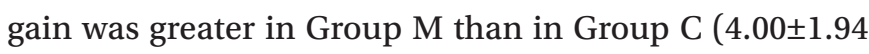

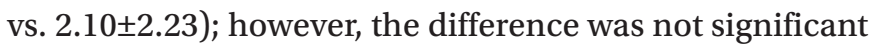
$(\mathrm{p}=0.106)$. Similarly, on comparing the MAL results between the two groups, we found that functional gain was greater in Group M than in Group C; however, the results for MAL-AOU $(0.17 \pm 0.17$ vs. $0.03 \pm 0.09)$ and for MALQOM $(0.04 \pm 0.07$ vs. $0.01 \pm 0.03)$ were not significantly different between the two groups ( $\mathrm{p}=0.092$ and $\mathrm{p}=0.273$, respectively) (Tables 4,5 ).

The pre- and post-treatment effects showed improvements; however, the differences were not statistically significant with regard to the FMA-UE and MAL scales.

The results of MP combined with conventional rehabilitation therapy and conventional rehabilitation therapy alone did not differ significantly on the FMA-UE and MAL scales.

Table 3. Comparison of the changes of FMA-UE between the $M+C$ phase and the $C$ phase

\begin{tabular}{|c|c|c|c|c|c|c|}
\hline \multirow{2}{*}{ FMA-UE } & \multicolumn{3}{|c|}{ Group 1} & \multicolumn{3}{|c|}{ Group 2} \\
\hline & Pre & Post & Change & Pre & Post & Change \\
\hline \multicolumn{7}{|l|}{$\mathrm{M}+\mathrm{C}$ phase } \\
\hline Patient \# 1 & 40 & 40 & 0 & 39 & 45 & 6 \\
\hline Patient \#2 & 45 & 49 & 4 & 47 & 50 & 3 \\
\hline Patient \#3 & 58 & 64 & 6 & 48 & 50 & 2 \\
\hline Patient \#4 & 55 & 59 & 4 & 42 & 47 & 5 \\
\hline Patient \#5 & 52 & 58 & 6 & 38 & 42 & 4 \\
\hline \multicolumn{7}{|l|}{$\mathrm{C}$ phase } \\
\hline Patient \# 1 & 40 & 48 & 8 & 36 & 39 & 3 \\
\hline Patient \#2 & 49 & 50 & 1 & 44 & 47 & 3 \\
\hline Patient \#3 & 64 & 65 & 1 & 47 & 48 & 1 \\
\hline Patient \#4 & 59 & 60 & 1 & 41 & 42 & 1 \\
\hline Patient \#5 & 58 & 59 & 1 & 37 & 38 & 1 \\
\hline
\end{tabular}

Data are derived by subtracting the values at the end of each phase from those at the start (T2 - T1, T1 - T0).

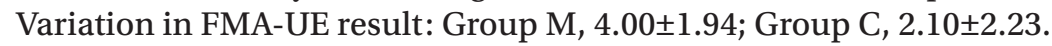

FMA-UE, Fugl-Meyer Assessment of upper extremity; M+C phase, mental practice and conventional therapy phase; C phase, conventional therapy phase only; Group $\mathrm{M}$, mental practice combined with conventional rehabilitation therapy; Group C, conventional rehabilitation therapy alone. 
Hyun Seung Oh, et al.

Table 4. Comparison of the changes in the MAL-AOU data between the $M+C$ phase and $C$ phase

\begin{tabular}{|c|c|c|c|c|c|c|}
\hline \multirow{2}{*}{ MAL-AOU } & \multicolumn{3}{|c|}{ Group 1} & \multicolumn{3}{|c|}{ Group 2} \\
\hline & Pre & Post & Change & Pre & Post & Change \\
\hline \multicolumn{7}{|l|}{$\mathrm{M}+\mathrm{C}$ phase } \\
\hline Patient \# 1 & 1.40 & 0.92 & 0.48 & 0.25 & 0.25 & 0.00 \\
\hline Patient \#2 & 0.80 & 0.42 & 0.38 & 3.80 & 4.00 & 0.20 \\
\hline Patient \#3 & 4.41 & 4.23 & 0.18 & 1.48 & 1.48 & 0.00 \\
\hline Patient \#4 & 4.50 & 4.21 & 0.29 & 1.80 & 2.00 & 0.20 \\
\hline Patient \#5 & 1.64 & 1.64 & 0.00 & 0.82 & 0.82 & 0.00 \\
\hline \multicolumn{7}{|l|}{ C phase } \\
\hline Patient \# 1 & 1.40 & 1.40 & 0.00 & 0.24 & 0.25 & 0.01 \\
\hline Patient \#2 & 0.80 & 0.80 & 0.00 & 3.51 & 3.80 & 0.29 \\
\hline Patient \#3 & 4.41 & 4.41 & 0.00 & 1.47 & 1.48 & 0.01 \\
\hline Patient \#4 & 4.50 & 4.50 & 0.00 & 1.80 & 1.80 & 0.00 \\
\hline Patient \#5 & 1.64 & 1.64 & 0.00 & 0.82 & 0.82 & 0.00 \\
\hline
\end{tabular}

Data are derived by subtracting the values at the end of each phase from those at the start $(\mathrm{T} 2-\mathrm{T} 1, \mathrm{~T} 1-\mathrm{T} 0)$.

Variation in MAL-AOU result: Group M, 0.17 \pm 0.17 ; Group C, 0.03 \pm 0.09 .

MAL-AOU, motor activity log amount of use; $\mathrm{M}+\mathrm{C}$ phase, mental practice and conventional therapy phase; $\mathrm{C}$ phase, conventional therapy phase only; Group $\mathrm{M}$, mental practice combined with conventional rehabilitation therapy; Group C, conventional rehabilitation therapy alone.

Table 5. Comparison of the changes in the MAL-QOM data between the $M+C$ phase and the $C$ phase

\begin{tabular}{|c|c|c|c|c|c|c|}
\hline \multirow{2}{*}{ MAL-QOM } & \multicolumn{3}{|c|}{ Group 1} & \multicolumn{3}{|c|}{ Group 2} \\
\hline & Pre & Post & Change & Pre & Post & Change \\
\hline \multicolumn{7}{|l|}{$\mathrm{M}+\mathrm{C}$ phase } \\
\hline Patient \# 1 & 0.60 & 0.60 & 0.00 & 0.00 & 0.00 & 0.00 \\
\hline Patient \#2 & 0.50 & 0.50 & 0.00 & 4.60 & 4.80 & 0.20 \\
\hline Patient \#3 & 4.20 & 4.20 & 0.00 & 1.23 & 1.28 & 0.05 \\
\hline Patient \#4 & 4.42 & 4.42 & 0.00 & 0.95 & 0.95 & 0.00 \\
\hline Patient \#5 & 1.34 & 1.48 & 0.14 & 0.00 & 0.00 & 0.00 \\
\hline \multicolumn{7}{|l|}{$\mathrm{C}$ phase } \\
\hline Patient \# 1 & 0.60 & 0.60 & 0.00 & 0.00 & 0.00 & 0.00 \\
\hline Patient \#2 & 0.50 & 0.50 & 0.00 & 4.60 & 4.60 & 0.00 \\
\hline Patient \#3 & 4.20 & 4.20 & 0.00 & 1.23 & 1.23 & 0.00 \\
\hline Patient \#4 & 4.42 & 4.50 & 0.08 & 0.95 & 0.95 & 0.00 \\
\hline Patient \#5 & 1.48 & 1.52 & 0.04 & 0.00 & 0.00 & 0.00 \\
\hline
\end{tabular}

Data are derived by subtracting the values at the end of each phase from those at the start (T2 - T1, T1 - T0).

Variation in MAL-QOM result: Group M, 0.04 \pm 0.07 ; Group C, $0.01 \pm 0.03$.

MAL-QOM, motor activity log quality of movement; M+C phase, mental practice and conventional therapy phase; C phase, conventional therapy phase only; Group $\mathrm{M}$, mental practice combined with conventional rehabilitation therapy; Group C, conventional rehabilitation therapy alone. 


\section{DISCUSSION}

We believe that this is the first MP study to include both 3D motion analysis and clinical assessments in stroke patients, with the same tasks being used for both evaluation methods and interventions. This study clinically evaluated the effects of adjuvant MP on upper limb function in stroke patients. In addition, this was an $\mathrm{AB} / \mathrm{BA}$ crossover study that allowed for observation of improvements in upper extremity function through 3D motion analysis when certain tasks identical to MP tasks were performed, such as drinking from a cup and opening a door; however, the improvements in the two conditions did not differ significantly.

With use of a crossover design, the same group of patients underwent both treatment sessions of interest in sequence and then in reverse order. Hence, we used randomization to determine the order in which the treatment sessions would be undertaken. The crossover design has some attractive features, that is, treatments could be compared 'within subjects' rather than 'between subjects' and only a small sample size was needed. However, such a two-period crossover trial has some important disadvantages. Because there might be a period effect or a carry-over of the treatment effect from one period to the next, we investigated the possibility of treatment-period interaction by assessing whether in the absence of an interaction, a patient's average response to the treatments would be the same regardless of the order in which the treatments were carried out. In addition, we investigated the possibility of a period effect by comparing the differences between the periods in the two groups of patients. Finally, we could assess the treatment effect on all 20 within-subject differences between the two treatments, because no period effect or treatment-period interaction was noted. The present study showed that there were improvements in upper limb function following a stroke in both Group M and Group C; however, no significant differences were noted between the groups.

In MP, it is important for participants to perform a task efficiently. In previous MP studies, either a good cognitive level on the MMSE (or modified MMSE) or the ability to understand and follow simple verbal instructions was the standard prerequisite for participation in this type of treatment $[1,8,10,11,15,16]$. Because there are no data for setting standards on whether patients can efficiently perform additional MP, we used the VMIQ to include patients who scored less than 2.26 with respect to adequate imagination skills for MP to enable effective upper extremity motor imagery $[17,18]$.

A previous study compared groups that performed MP for a duration of 20,40 , or 60 minutes [10]. No definite change patterns were noted with regard to functional limitation of the impaired arm, indicating that repetitive, task-specific practice was effective when MP was combined, regardless of 'dose' level. Therefore, the patients in our study performed MP for 20 minutes at a time, taking into consideration the factors of compliance and fatigue.

The majority of previous studies of MP used tasks such as reaching for and grasping a cup or object, turning a page in a book, writing with a pen/pencil, eating, and using a hairbrush or comb. However, because functional assessments of the upper extremity involved the use of FMA-UE, ARAT, and MAL as tools for analysis, the training data used in MP and the evaluation results in these studies showed a mismatch, making changes in ADLs difficult to observe. Therefore, with 3D motion analysis, we aimed to observe real changes in the patient's actions by assessing the activities mentioned in MP training methods, including simple daily tasks, such as drinking from a cup and opening a door.

A previous study that targeted subjects who had had a stroke within the previous 1 to 3 months and who practiced MP and conventional therapy simultaneously noted improvements in the Motricity Index on the upper extremity and arm functional test [1]. Similarly, another study showed that in targeted groups with subacute stroke (i.e., a stroke that occurred within the previous 6 months), improvement was greater with MP combined with conventional therapy than with conventional therapy alone [21]. However, in our study, the MP group showed some improvement in upper extremity function, although there was little difference between the groups. These results correspond to those from a report claiming that patients with subacute stroke did not benefit from MP in terms of increasing motor recovery [12].

Although motor imagery might be beneficial for the recovery of motor function after a stroke, limited information is available on the MP protocol for motor neurorehabilitation. We were primarily interested in therapeutic efficacy during 3D motion in real life.

The findings reported here might be of value consid- 
ering that we used 3D motion analysis during MP to objectively assess everyday tasks, such as drinking from a cup and opening a door. In addition, our study differs from existing studies in that the unbiased evaluation of qualities of movement (e.g., ROM, joint motion torque, velocity, and motion smoothness) provided observable changes in function before and after MP by means of objective 3D motion analysis. We compared the results of this analysis with those obtained using conventional assessment tools (FMA-UE and MAL), and in all cases the corresponding findings were negative.

Based on the hypothesis that neural loops and movement patterns are activated, these clinical effects might imply that MP alone can achieve the same results as those obtained when performing the actual tasks [1]. This conclusion was supported by a study in which changes in cerebral function that occurred after MP were the same as those seen with actual training [7]. In another supporting study, MP activated motor-related brain lesions and induced cortical plasticity [8].

The present study had some limitations, one of which was the small number of patients tested. Although we showed no interactions in the period effect and treatment period, MP performed along with conventional therapy did show positive results, albeit the differences between the groups were not statistically significant. We found that a relatively large standard deviation might have resulted in negative results. Even if our study had been designed to exclude the period effect initially, spontaneous functional recovery from subacute stroke would most likely have occurred. In addition, although we hoped to overcome 'consciousness factors', such as the patient's attention and concentration during MP, differing levels of compliance could have affected our results. The patients were asked to perform MP for 20 minutes because a previous study has shown that repetitive, task-specific practice was efficient when MP was combined, regardless of the duration. However, in a recent study, superior results were obtained with high-dose MP than with low-dose MP. Considering this recent finding, the short duration of MP in our study might have been less effective [22].

To rule out the possibility of spontaneous recovery, we did not include a control group (i.e., no treatment) in the comparison; therefore, a controlled study with a larger sample size may be required in the future.

Our primary interest in this study was to determine the effect of MP in patients with stoke during 3D motion in real life. The results using clinical assessment tools as well as 3D motion analysis indicated that adjuvant MP had no significant effect on post-stroke function of the affected upper limb. This study is important in that we used objective 3D motion analysis to evaluate the effect of MP in this group of patients. Furthermore, such an analysis could assess the tasks and the evaluative tools and measure actual changes in movement. Nevertheless, future studies will be needed to verify the validity and reliability of the objectivity of 3D motion analysis. Based on the findings reported here, we expect 3D motion analysis to be used as an objective tool for assessing upper limb function in patients who have had a stroke. Future studies with larger sample sizes are required to validate the effects of MP.

\section{CONFLICT OF INTEREST}

No potential conflict of interest relevant to this article was reported.

\section{ACKNOWLEDGMENTS}

The 3D motion analysis for this study was performed by Pil-Woo Hwang of the Korea National Rehabilitation Research Institute, Seoul, Korea.

\section{REFERENCES}

1. Riccio I, Iolascon G, Barillari MR, Gimigliano R, Gimigliano F. Mental practice is effective in upper limb recovery after stroke: a randomized single-blind crossover study. Eur J Phys Rehabil Med 2010;46:19-25.

2. Mulder T, Zijlstra S, Zijlstra W, Hochstenbach J. The role of motor imagery in learning a totally novel movement. Exp Brain Res 2004;154:211-7.

3. Cicinelli P, Marconi B, Zaccagnini M, Pasqualetti P, Filippi MM, Rossini PM. Imagery-induced cortical excitability changes in stroke: a transcranial magnetic stimulation study. Cereb Cortex 2006;16:247-53.

4. Barclay-Goddard RE, Stevenson TJ, Poluha W, Thalman L. Mental practice for treating upper extremity deficits in individuals with hemiparesis after stroke. Cochrane Database Syst Rev 2011;(5):CD005950.

5. Malouin F, Jackson PL, Richards CL. Towards the inte- 
gration of mental practice in rehabilitation programs: a critical review. Front Hum Neurosci 2013;7:576.

6. Lacourse MG, Turner JA, Randolph-Orr E, Schandler SL, Cohen MJ. Cerebral and cerebellar sensorimotor plasticity following motor imagery-based mental practice of a sequential movement. J Rehabil Res Dev 2004;41:505-24.

7. Jackson PL, Lafleur MF, Malouin F, Richards CL, Doyon J. Functional cerebral reorganization following motor sequence learning through mental practice with motor imagery. Neuroimage 2003;20:1171-80.

8. Page SJ, Szaflarski JP, Eliassen JC, Pan H, Cramer SC. Cortical plasticity following motor skill learning during mental practice in stroke. Neurorehabil Neural Repair 2009;23:382-8.

9. Braun S, Kleynen M, van Heel T, Kruithof N, Wade D, Beurskens A. The effects of mental practice in neurological rehabilitation; a systematic review and metaanalysis. Front Hum Neurosci 2013;7:390.

10. Page SJ, Dunning K, Hermann V, Leonard A, Levine P. Longer versus shorter mental practice sessions for affected upper extremity movement after stroke: a randomized controlled trial. Clin Rehabil 2011;25:627-37.

11. Page SJ, Levine P, Leonard A. Mental practice in chronic stroke: results of a randomized, placebocontrolled trial. Stroke 2007;38:1293-7.

12. Ietswaart M, Johnston M, Dijkerman HC, Joice S, Scott CL, MacWalter RS, et al. Mental practice with motor imagery in stroke recovery: randomized controlled trial of efficacy. Brain 2011;134(Pt 5):1373-86.

13. Crosbie JH, McDonough SM, Gilmore DH, Wiggam MI. The adjunctive role of mental practice in the rehabilitation of the upper limb after hemiplegic stroke: a pilot study. Clin Rehabil 2004;18:60-8.
14. Dijkerman HC, Ietswaart M, Johnston M, MacWalter RS. Does motor imagery training improve hand function in chronic stroke patients? A pilot study. Clin Rehabil 2004;18:538-49.

15. Page SJ, Murray C, Hermann V, Levine P. Retention of motor changes in chronic stroke survivors who were administered mental practice. Arch Phys Med Rehabil 2011;92:1741-5.

16. Page SJ, Levine P, Leonard AC. Effects of mental practice on affected limb use and function in chronic stroke. Arch Phys Med Rehabil 2005;86:399-402.

17. Choi YI. The effects of mental practice for performing of functional activities to unilateral neglect and ADL in person with stroke. J Korea Acad Ind Coop Soc 2009;10:3879-87.

18. Isaac A, Marks DF, Russell DG. An instrument for assessing imagery of movement: the Vividness of Movement Imagery Questionnaire (VMIQ). J Ment Imag 1986;10:23-30.

19. Hingtgen B, McGuire JR, Wang M, Harris GF. An upper extremity kinematic model for evaluation of hemiparetic stroke. J Biomech 2006;39:681-8.

20. Alt Murphy M, Willen C, Sunnerhagen KS. Kinematic variables quantifying upper-extremity performance after stroke during reaching and drinking from a glass. Neurorehabil Neural Repair 2011;25:71-80.

21. Muller K, Butefisch CM, Seitz RJ, Homberg V. Mental practice improves hand function after hemiparetic stroke. Restor Neurol Neurosci 2007;25:501-11.

22. Pollock A, Farmer SE, Brady MC, Langhorne P, Mead GE, Mehrholz J, et al. Interventions for improving upper limb function after stroke. Cochrane Database Syst Rev 2014;11:CD010820. 